A. Bayo-Salas, J. Beerten, J. Rimez and D. Van Hertem, "Impedance-based stability assessment of parallel VSC HVDC grid connections," Proc. IET International Conference on $A C$ and DC Power Transmission ACDC 2015, 11th ed., Birmingham, UK, Feb. 10-12, 2015, 9 pages.

Digital Object Identifier: 10.1049/cp.2015.0087

URL (IET Digital Library):

http://digital-library.theiet.org/content/conferences/10.1049/cp.2015.0087

URL (IEEE Xplore Digital Library):

http://ieeexplore.ieee.org/xpl/articleDetails.jsp?arnumber=7140569

(C) 2015 IET. This paper is a postprint of a paper submitted to and accepted for publication in Proc. IET International Conference on AC and DC Power Transmission 2015 and is subject to Institution of Engineering and Technology Copyright. The copy of record is available at IET Digital Library. 


\title{
Impedance-based stability assessment of parallel VSC HVDC grid connections
}

\author{
A. Bayo-Salas ${ }^{* \dagger}$, J. Beerten ${ }^{\dagger \dagger}$, J. Rimez ${ }^{*}$, D. Van Hertem ${ }^{\dagger}$ \\ *Elia System Operator, Belgium, alejandro.bayosalas@elia.be, ${ }^{\dagger}$ ELECTA, KU Leuven, Belgium, $\ddagger N T N U$, Norway
}

Keywords: Stability study, control interactions, VSC HVDC, Impedance-based analysis

\begin{abstract}
The number of converters connected to the system is likely to be increased. These devices may interact with each other through the network. TSOs are concerned about the impact of these interactions and its influence on the stability. This paper presents the stability analysis of a system with two converters. The problem is studied in the frequency domain by using an impedance-based approach. A model for assessing the closed-loop stability of the complete system is developed. This approach shows to have potential for studying the interactions in larger networks. The relative stability under different grid parameters is addressed. Simulation results show that the stability is compromised when a parallel converter is connected.
\end{abstract}

\section{Introduction}

Current investments in power generation are encouraging the installation of offshore wind farms in the North Sea. In parallel, grid planning and market integration are driving the need for more interconnection between countries. High Voltage Direct Current (HVDC) lines are seen as the preferred solution for accommodating the increase in renewable energies and additional interconnection capacity due to their controllability and adequacy for long underground and undersea connections. The technology used for the connection of the converter-based wind generators and transferring power through HVDC lines is the voltage source converter (VSC). As future planning for the extension to an offshore grid, increasing number of VSCs will be closely connected to each other at the AC side. These VSCs, which might be built by different manufacturers, could impact on the operation of each other. The latter gives rise to crucial concerns such as the influence of one converter in other devices and its range of impact. One of the outstanding research questions is the study of possibly adverse control interactions between such converters.

When a new converter is connected to the grid, it has an influence on the AC network characteristics. The grid will change towards a system increasingly dominated and hence influenced by such devices. These converters are commonly designed for operating independently, i.e., without considering the impact of future connected converters. Currently the VSC control design is mainly considering the simplification of the grid response at the Point of Common Coupling (PCC). However the VSC might interact with other converters leading to a poor response and results in stability concerns and more uncertainties during the design phase. As a result, connection studies for such converters might require considering the characteristics and parameters of VSCs closely connected. Such a future scenario will thus require a continuous study and the flexibility of the converters to be adapted to the new conditions and requirements as well as urges for a better knowledge in order to anticipate future possible issues.

Lately the subject of interactions between converters is gaining a lot of interest and triggers some outstanding research questions. First the influence of the integration of a new VSC in the operation of the rest of connected converters is unknown. Second the concept of electrical proximity or the range of these adverse interactions is unknown and relevant. Based on this proximity of influence, stability studies will consider or neglect the impact of a determined converter and hence adding complexity or reducing the model to be used in the evaluation.

The integration of these devices in the AC grid involves several studies with high complexity and requires a deep interdisciplinary knowledge. Harnefors et al. [1] used the promising impedance-based approach in stability studies for VSC-based systems. In the reference the VSC has been found to add an equivalent negative resistance at the PCC and thereby thus degrades the damping of the overall system. Interactions within the control bandwidth cause power quality and stability issues [2-4]. Subsynchronous interactions between a VSC and a nearby synchronous generator are addressed with the impedance-based approach in [5]. The same methodology is used for evaluating the stability in a VSC-based infeed connection [6]. Kocewiak et al.[4] applied frequency methods to study the impact of wind farm components and number of turbines on the stability and interferences at the collector point. Control interactions between a VSC and a STATCOM connected at the same point in steady-state are studied in [7]. An harmonic stability study with the impedance-based approach in an islanded system composed by three converters is addressed in [8].

However interactions between VSCs connected at different points in the grid have not been studied so far. The role of the 


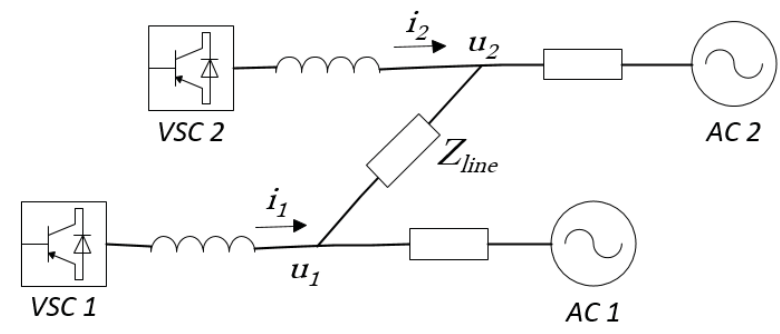

Fig. 1: System with two parallel-connected VSCs.

network interconnecting them and the impact of the distance on the interactions is not clear.

This paper investigates the interactions between two converters connected at different points of the grid. For doing so, the problem is studied in the frequency domain as a Multi-Input Multi-Output (MIMO) system. The closed-loop response of this coupled system is evaluated. The main contribution of this paper is to present an analytical methodology for studying the closed-loop interactions in the frequency domain. This will allow to study the degree of coupling and/or potential issues in future connections by means of impedance models of the different components, in an easily adaptable way by only changing the frequency-dependant impedances and without the limitation of only relying on time-consuming EMT simulation studies for a large number of scenarios or highly complex and large state-space matrices.

\section{Model of the AC network with parallel-connected converters}

The system in study is shown in Figure 1. It consists of two converters connected to the AC system at different PCCs and interconnected through a transmission line.

The problem is studied in the frequency domain which allows to give a quick and simple check to conditions which give rise to stability issues. The whole network is transformed into an equivalent circuit. The elements which compose this system are converted into the reciprocal circuit equivalent models as explained in the section. The VSC is transformed into a current source behind an admittance, which characterizes the VSC frequency response, AC networks are converted into voltage sources behind an equivalent impedance and the transmission line is calculated as the impedance seen from the respective PCC. The equivalent circuit of the studied system is depicted in Figure 2.

Next the modelling of the different components is detailed.

\subsection{Input-admittance modelling of the VSC}

A way to characterize the converter dynamics is to consider the converter as a frequency-dependant admittance seen from the PCC. The representation of the VSC as an input-admittance

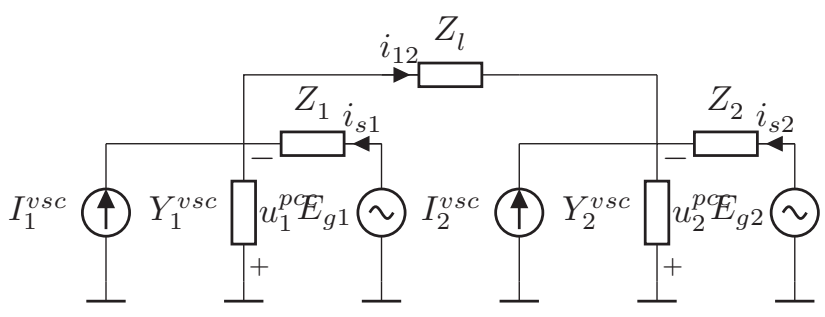

Fig. 2: Equivalent circuit of the system.

has become generalized and widely used in stability studies [1,5-6,8-10]. In this methodology, the converter is represented by its Norton equivalent circuit as a current-controlled source through an admittance as seen in Figure 2. The admittance $Y_{v s c}$ characterizes the frequency response of the reactor, commutation of switching devices and its control strategy and parameters. The input-admittance model allows to study the response in the frequency-domain.

The injected controlled current to the PCC is with this approach hence obtained as function of the external set-points and the PCC voltage $u^{s}$ regarded as the disturbance,

$$
i_{v s c}=G(s) \cdot i^{r e f}+Y_{v s c}(s) u^{s}
$$

where $G(s)$ is the outer loop and $i^{r e f}$ represent the external set-point.

The equivalent admittance can be derived in two ways. One consists on scanning the harmonic response from the converter at the connection point. By this, non-linearities of switching devices and the harmonic generation can be represented. The other consists on analytically modelling the response in the frequency domain. This solution is based on the derivation of the ordinary differential equations which define the model. Since the system is non-linear, the linearisation around one operating point is performed and thus it is only valid in the close proximity to this operation point. In the paper, the equivalent admittance is analytically obtained from a general and basic model.

$Y_{v s c}$ consists of a transfer function from the input voltage $u^{s}$ to the output current $i$. Since equations and control are implemented into the synchronous $d q$ frame, the admittance $Y_{v s c}$ is a matrix of TFs composed by the diagonal elements relating $d$ and $q$ components respectively and the couplings in the non-diagonal terms as,

$$
\left(\begin{array}{c}
\Delta i_{d} \\
\Delta i_{q}
\end{array}\right)=\left(\begin{array}{cc}
Y_{v s c}^{d d} & Y_{v s c}^{d q} \\
Y_{v s c}^{q d} & Y_{v s c}^{q q}
\end{array}\right)\left(\begin{array}{c}
\Delta u_{d}^{s} \\
\Delta u_{q}^{s}
\end{array}\right)
$$

The control is implemented in the $d q$ reference frame. The VSC model is depicted in Figure 3. The basic control principle for the VSC is based on a two-level cascaded control. Active and reactive power are independently controlled. Since the study is only focused on the dynamics in the AC system, the DC link voltage is considered constant and thus the dynamics from the DC side are not considered in this paper.

The AC grid dynamics in the rotatory frame are given by, 


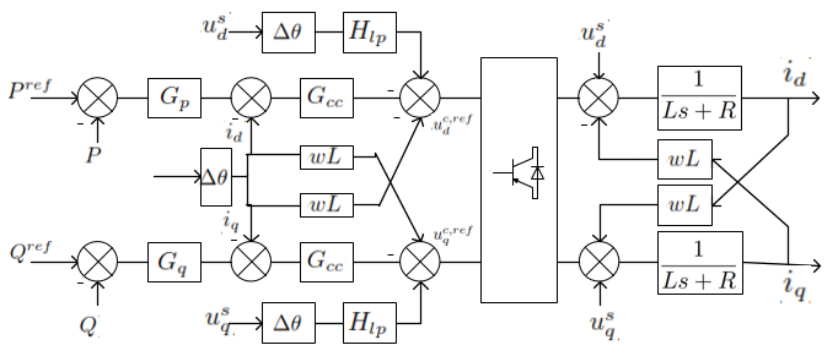

Fig. 3: VSC model ans control.

$$
R\left(\begin{array}{l}
i_{d} \\
i_{q}
\end{array}\right)+L \frac{d}{d t}\left(\begin{array}{l}
i_{d} \\
i_{q}
\end{array}\right)=\left(\begin{array}{l}
u_{d}^{s} \\
u_{q}^{s}
\end{array}\right)-\left(\begin{array}{l}
u_{d}^{c} \\
u_{q}^{c}
\end{array}\right)+\left(\begin{array}{c}
-\omega L i_{q} \\
\omega L i_{d}
\end{array}\right)
$$

where $R+L s$ is the VSC reactor, from this point on $X_{r}, u_{d q}^{s}$ are the voltages at the PCC and $u_{d q}^{c}$ the modulated voltages at the VSC terminals. The inner current control provides the voltage references $u_{d q}^{c, r e f}$ sent to the switching valves,

$$
\begin{aligned}
\left(\begin{array}{c}
\Delta u_{d}^{c, r e f} \\
\Delta u_{q}^{c, r e f}
\end{array}\right)= & -\left(\begin{array}{c}
G_{c c}^{d}(s) \\
G_{c c}^{q}(s)
\end{array}\right)\left(\begin{array}{c}
\Delta i_{d}^{r e f}-\Delta i_{d} \\
\Delta i_{q}^{r e f}-\Delta i_{q}
\end{array}\right) \\
& +w L\left(\begin{array}{c}
-\Delta i_{q} \\
\Delta i_{d}
\end{array}\right)+\left(\begin{array}{c}
\Delta \hat{u}_{d}^{s} \\
\Delta \hat{u}_{q}^{s}
\end{array}\right)
\end{aligned}
$$

where $\hat{u}_{d q}^{s}$ are the measured grid voltages and $G_{c c}$ represents a PI controller,

$$
G_{c c}(s)=K_{p}^{c c}+\frac{K_{i}^{c c}}{s}
$$

Noise and harmonics in the measured grid voltage, $\hat{u}_{d q}^{s}$, are attenuated through a low-pass filter $H_{l p}$ for a cut-off frequency $\alpha_{f f}$ in order to improve the harmonic rejection capability of the current controller.

$$
\Delta \hat{u}_{d q}^{s}=\frac{\alpha_{f f}}{s+\alpha_{f f}} \Delta u_{d q}^{s}
$$

The PWM switching delay $D_{\text {pwm }}$, shown as the converter in Figure 3, from $u_{d q}^{c, r e f}$ to $u_{d q}^{c}$ is modelled as a first-order Padé approximation.

$$
D_{p w m}(s)=\frac{1}{t_{p w m} s+1}
$$

Equation (4) is substituted in (3) and its terms are arranged by considering $u_{d q}^{s}$ and $i_{d q}^{r e f}$ as the inputs and $i_{d q}$ as the output. The transfer functions of the inner controller and AC dynamics are given by, ${ }^{1}$

$$
i_{d q}=\underbrace{\left(\frac{1-D_{p w m} H_{l p}}{X_{r}+D_{p w m} G_{c c}}\right)}_{X_{c c}} u_{d q}^{s}+\underbrace{\left(\frac{D_{p w m} G_{c c}}{X_{r}+D_{p w m} G_{c c}}\right)}_{H_{c c}} i_{d q}^{r e f}
$$

In the matrix form,

$$
i_{d q}=\left(\begin{array}{cc}
X_{c c} & 0 \\
0 & X_{c c}
\end{array}\right) u_{d q}^{s}+\left(\begin{array}{cc}
H_{c c} & 0 \\
0 & H_{c c}
\end{array}\right) i_{d q}^{r e f}
$$

\footnotetext{
${ }^{1}$ From now on the term $s$ in TFs as function of Laplace operator is neglected due to redundancy.
}

where $X_{c c}$ is the VSC equivalent admittance only considering the current controller and reactor and $H_{c c}$ is the transfer function from the current reference to $i_{d q}$.

The disturbances from the coupled $d q$ currents are compensated for by considering a correct decoupling in the control. The $d q$ reference currents in Equation (4) are computed by the outer loops which track the active and reactive power set-point, $P^{r e f}$ and $Q^{r e f}$, respectively,

$$
\begin{aligned}
& i_{d}^{r e f}=G_{p}\left(P^{r e f}-P\right) \\
& i_{q}^{r e f}=G_{q}\left(Q^{r e f}-Q\right)
\end{aligned}
$$

where $G_{p}$ and $G_{q}$ are the PI controls of the active and reactive power controller respectively.

Active and reactive powers are measured using a second-order low-pass filter $F_{2 n d}$,

$$
F_{2 n d}=\frac{\omega_{0}^{2}}{s^{2}+2 \omega_{0} \xi s+\omega_{0}^{2}}
$$

The implemented active power control for the $d$ component is shown in Figure 4. Equations $\left(P=u_{d}^{s} i_{d}+u_{q}^{s} i_{q}\right.$ and $Q=-u_{d}^{s} i_{q}+u_{q}^{s} i_{d}$ ) introduce non-linearities and thus the dynamic model is linearised around the operating point $\left(i_{d 0}=\frac{P_{0}}{u_{d 0}^{s}}, i_{q 0}=-\frac{Q_{0}}{u_{d 0}^{s}}\right)$ as,

$$
\begin{aligned}
& \Delta P=F_{2 n d} \frac{P_{0}}{u_{d 0}^{s}} \Delta u_{d}^{s}+F_{2 n d} u_{d 0}^{s} \Delta i_{d}+F_{2 n d} \frac{Q_{0}}{u_{d 0}^{s}} \Delta u_{q}^{s} \\
& \Delta Q=F_{2 n d} \frac{P_{0}}{u_{d 0}^{s}} \Delta u_{q}^{s}-F_{2 n d} u_{d 0}^{s} \Delta i_{q}+F_{2 n d} \frac{Q_{0}}{u_{d 0}^{s}} \Delta u_{d}^{s}
\end{aligned}
$$

Then active and reactive reference currents in Equations (10) and (11) are derived (considering $P^{r e f}$ and $Q^{r e f}$ as constants) and linearised in the form,

$$
\begin{aligned}
& \Delta i_{d}^{r e f}=-G_{p}(\Delta P) \\
& \Delta i_{q}^{r e f}=-G_{q}(\Delta Q)
\end{aligned}
$$

By substituting Equations (15), (16), (13) and (14) in the equivalent admittance in Equation (8), the outer loops are included as,

$$
\left(\begin{array}{c}
\Delta i_{d} \\
\Delta i_{q}
\end{array}\right)=\left(\begin{array}{cc}
X_{c c}-H_{c c} G_{p} \frac{P_{0}}{u_{d 0}^{s}} & \frac{H_{c c} G_{p} \frac{Q_{0}}{u_{d 0}^{s}}}{1+H_{c c} G_{p} F_{2 n d} u_{d 0}^{s}} \\
\frac{-H_{c c} G_{q} \frac{Q_{0}}{u_{d 0}^{s}}}{1+H_{c c} G_{p} F_{2 n d} u_{d 0}^{s}} & \frac{X_{c c}-H_{c c} G_{q} \frac{P_{0}^{s}}{u_{d 0}^{s}}}{1-H_{c c} G_{q} F_{2 n d} u_{d 0}^{s}}
\end{array}\right)\left(\begin{array}{l}
\Delta u_{d}^{s} \\
\Delta u_{q}^{s}
\end{array}\right)
$$

Note that after the addition of outer loops and the linearisation, non-diagonal elements appear in the matrix. The $i j$ terms in Equation (17) are denominated as,

$$
\left(\begin{array}{c}
\Delta i_{d} \\
\Delta i_{q}
\end{array}\right)=\left(\begin{array}{ll}
G_{d d} & G_{d q} \\
G_{q d} & G_{q q}
\end{array}\right)\left(\begin{array}{c}
\Delta u_{d}^{s} \\
\Delta u_{q}^{s}
\end{array}\right)
$$

The PLL has been included in the model using the approach presented in [1]. 


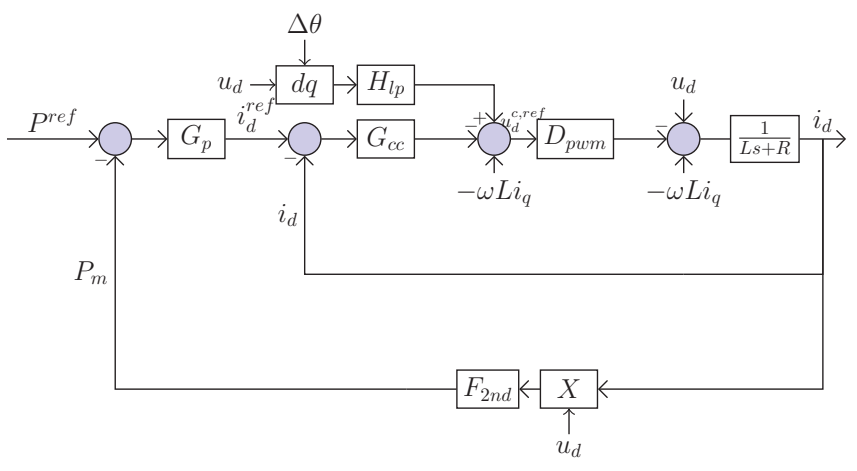

Fig. 4: $d$-component loop for the active power.

The matrix of closed-loop input-admittance transfer functions $Y_{V S C}$ is finally given by,

$$
\begin{gathered}
Y_{v s c}^{d d}=G_{d d} \\
Y_{v s c}^{d q}=G_{d q}-\left(\frac{Q_{0}}{u_{d 0}}\right) G_{p l l} \\
Y_{v s c}^{q d}=G_{q d} \\
Y_{v s c}^{q q}=G_{q q}\left(1-u_{d 0}^{s} G_{p l l}\right)+\left(\frac{P_{0}}{u_{d 0}^{s}}\right) G_{p l l}
\end{gathered}
$$

where $G_{p l l}$ is,

$$
G_{p l l}=\frac{C_{p l l}}{s+u_{d 0}^{s} C_{p l l}}
$$

and $C_{p l l}$ is the PLL PI controller.

The VSC input-admittance is modelled with the parameters shown in Table 1.

Admittance magnitude and phase angle are plotted in Figures

\begin{tabular}{|l|l|}
\hline VSC parameters & Values \\
\hline$S_{n}$ & $1000 \mathrm{MVA}$ \\
$V_{n}$ & $380 \mathrm{kV}$ \\
Reactor & $0.0036+0.18 s[\mathrm{pu}]$ \\
Inner control & $G_{c c}=22.5+\frac{0.45}{s}$ \\
$P$ control & $G_{p}=0.10+\frac{12.5}{s}$ \\
$Q$ control & $G_{q}=0.10+\frac{12.5}{s}$ \\
$H_{l p}$ & $\alpha_{f f}=20 \mathrm{~Hz}$ \\
$F_{2 n d}$ & $\omega_{0}=140 \mathrm{~Hz} \xi=0.7$ \\
$t_{s h}$ & $0.5 \mathrm{~ms}$ \\
\hline
\end{tabular}

Table 1: VSC control parameters.

5 and 6 respectively. The passivity of a system, or the positive resistance of an electrical component, is defined by the region with a phase angle between -90 and $90 \mathrm{deg}$. Such response is seen in Figure 6 where the angle is within this range above 2 Hz. The angle is lagging -90 deg when $\omega \rightarrow \infty$ due to the inductive behaviour of the converter. The negative-conductance region is in this case situated in the frequency range under 2 $\mathrm{Hz}$.

Negative-conductance regions indicate frequencies at which the converter is seen as a negative damping from the system. Thus the converter may cause oscillations at those frequencies to grow.

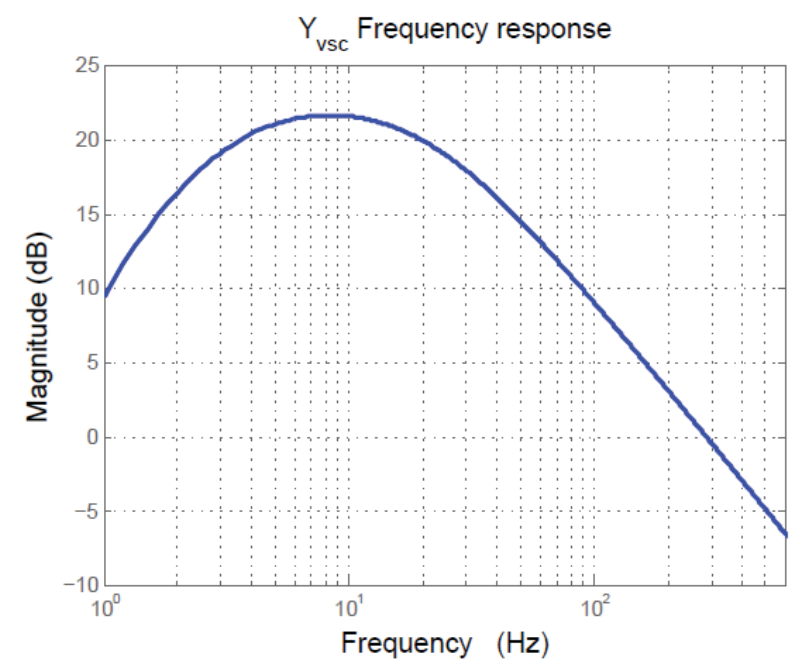

Fig. 5: Magnitude of the equivalent VSC admittance $Y_{v s c}$.

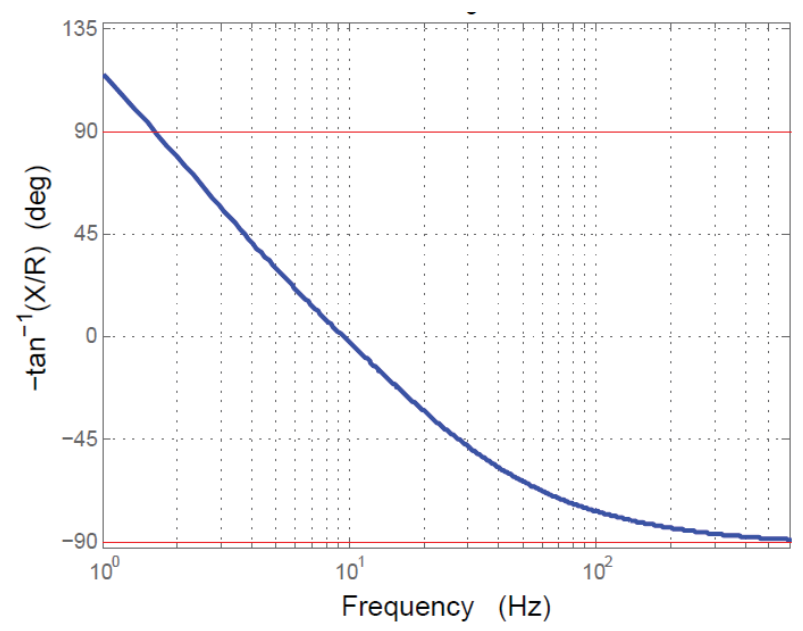

Fig. 6: Phase angle of the equivalent VSC admittance $Y_{v s c}$.

Different modifications in the control such as parameters, additional filtering or use of other strategies will affect the frequency response of the converter and thus its interaction with the system. However the influence of these parameters in the interactions is not studied in the paper.

\subsection{AC network}

One of the most basic and simple ways of modelling the AC network is by means of representing it by a voltage source behind an impedance $Z_{a c}$. $Z_{a c}$ is a series RL representing the short-circuit impedance at the node, which is an approximation of the system strength at the PCC.

The short-circuit impedance, $Z_{s c}$ is defined by the quotient $\frac{|V|^{2}}{S_{s c}}$, where $S_{s c}$ is the short-circuit power. The equivalent resistance $R_{g}$ and inductance $L_{g}$ of the Thevenin model of the network are obtained from the magnitude of the impedance and 
the angle defined by the $X / R$ ratio which defines the quotient between the reactance and resistance of the network.

Since the analysis is performed in the $d q$ frame, all the network side dynamics are converted into the rotatory frame being the resulting matrix equal to,

$$
Z_{A C}(s)=\left(\begin{array}{cc}
Z_{A C}^{d d} & Z_{A C}^{d q} \\
Z_{A C}^{q d} & Z_{A C}^{q q}
\end{array}\right)
$$

where the $i, j$ elements of the matrix are,

$$
\begin{gathered}
\mathrm{Z}_{A C}^{d d}=Z_{A C}^{q q}=R_{g}+s \cdot L_{g} \\
Z_{A C}^{d q}=-Z_{A C}^{q d}=-\omega L_{g}
\end{gathered}
$$

\subsection{AC transmission line}

In the paper switching and modulation transients are not considered and thus neither the resulting harmonics at those frequencies. Due to the focus on the frequencies around the fundamental and specially at lower values covering the bandwidth of the control system, the equivalent $\pi$ model of the transmission line with lumped parameters is considered to be accurate. The series impedance and shunt admittance are equal to,

$$
\begin{gathered}
Z_{\text {series }}=R+j X[\Omega / \mathrm{km}] \\
Y_{\text {shunt }}=\jmath \omega C[\mathrm{~S} / \mathrm{km}]
\end{gathered}
$$

\begin{tabular}{|l|l|}
\hline $\begin{array}{l}\pi \text { param- } \\
\text { eters }\end{array}$ & Values \\
\hline$R$ & $29[\mathrm{~m} \Omega / \mathrm{km}]$ \\
$X$ & $0.32[\Omega / \mathrm{km}]$ \\
$\frac{\omega C}{2}$ & $1.9[\mu \mathrm{S} / \mathrm{km}]$ \\
\hline
\end{tabular}

Table 2: Parameters of the transmission line $\pi$ model .

\section{Interactions between parallel-connected converters}

As a common practice in the VSC control design, the AC dynamics are simplified by an equivalent network at the PCC and hence parallel influences with other electronic devices are often neglected. When two converters are being integrated at different points of the grid and following the standard approach, the control design only considers the characteristics at the PCC. Thus both converters would operate correctly under the assumption of an independent operation. However unexpected or unconsidered mutual couplings between both converters might lead to a poor response or pose a threat to the stability of the system because of interactions between them. In this section, the methodology for studying the interactions is developed and explained. To that end, a MIMO system composed by two Single-Input Single-Output (SISO) systems is formulated. Each of the SISO systems corresponds to one VSC. Their couplings are function of the network impedances

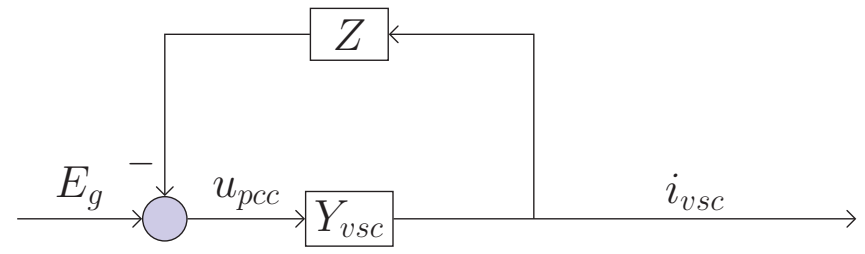

Fig. 7: Closed-loop response of the input-admittance with the grid impedance.

of the circuit. The complete system is sketched in Figure 8.

But before tackling the closed-loop response of this system, the stability study of a VSC connected to the AC grid with an impedance-based approach is introduced.

The open-loop response of the converter is defined by,

$$
i_{v s c}=G \cdot i_{r e f}+Y_{v s c} u_{p c c}
$$

When the converter is integrated into the system, the controlled current is injected to the $\mathrm{AC}$ grid through an impedance which distorts the PCC voltage. The disturbance is also propagated through the VSC control by means of the feed-forward voltage. This voltage disturbance is defined by,

$$
u_{p c c}=E_{g}-Z \cdot i_{v s c}
$$

Then the closed-loop response is indicated by the feedback depicted in Figure 7 and calculated from Equations (26) and (27) as,

$$
i_{v s c}=\frac{G}{1+Z Y_{v s c}} i_{r e f}+\frac{Y}{1+Z Y_{v s c}} E_{g}
$$

This model is extended to the system depicted in Figure 2 with two converters. Hence the current injected at the PCC from the VSC modelled as a current source with the admittance in parallel is,

$$
i_{v s c, k}=I_{v s c, k}+Y_{v s c, k} u_{p c c, k} \quad k=1,2
$$

The PCC voltage is defined as,

$$
u_{p c c, k}=E_{g k}-Z_{k} i_{s, k}
$$

where $i_{s, k}$ is the current injected to the AC grid equal to $i_{s, k}=$ $i_{v s c, k}+i_{12}$ and the current through the line $i_{12}$ is, ${ }^{2}$

$$
i_{12}=Z_{l}\left(u_{p c c, 1}-u_{p c c, 2}\right)
$$

Substituting (30) into (31), the PCC voltages are obtained as function of the Thevenin voltages and the output currents as,

$$
\begin{array}{r}
u_{p c c 1}=\left(1-\frac{Z_{1}}{Z_{\Sigma}}\right) E_{g 1}+\left(\frac{Z_{1}^{2}}{Z_{\Sigma}}-Z_{1}\right) i_{v s c 1} \\
+\frac{Z_{1}}{Z_{\Sigma}} E_{g 2}-\frac{Z_{1} Z_{2}}{Z_{\Sigma}} i_{v s c 2}
\end{array}
$$

\footnotetext{
2 The transmission line is considered symmetrical in the paper. Thus impedances $Z_{l}$ seen at both ends are equal. Otherwise equations must consider the respective impedance seen at the point of study.
} 


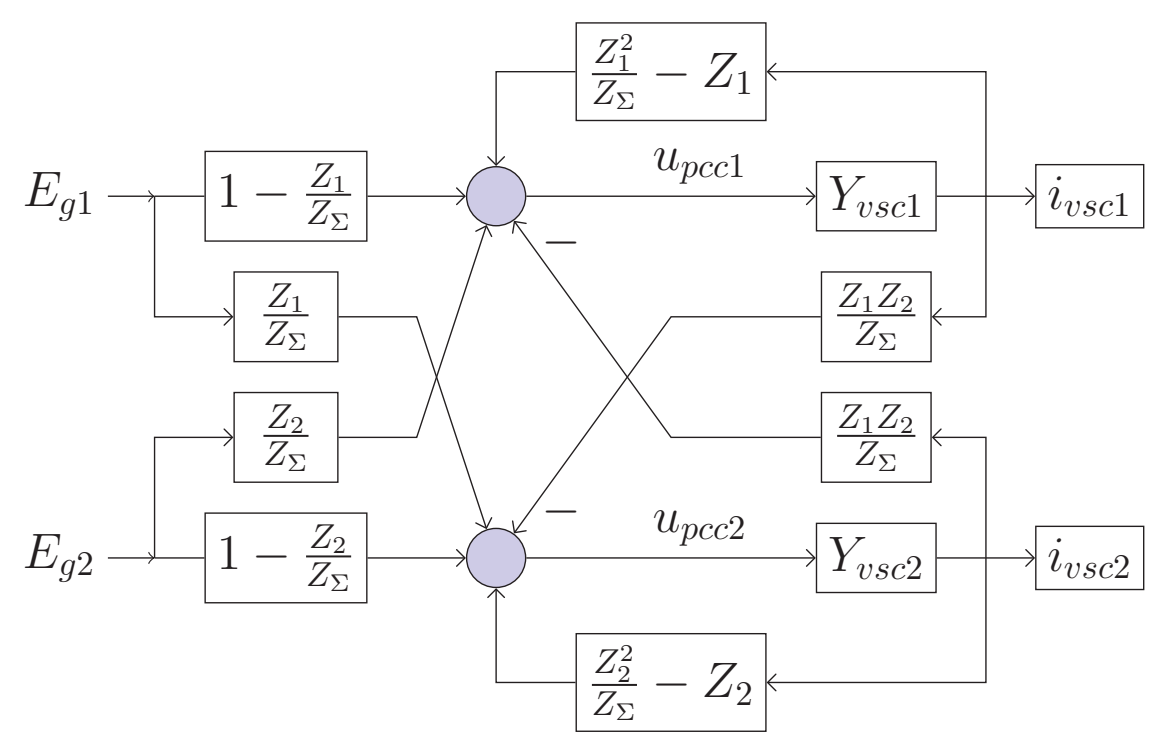

Fig. 8: MIMO system of interacting parallel-connected converters.

$$
\begin{array}{r}
u_{p c c 2}=\left(1-\frac{Z_{2}}{Z_{\Sigma}}\right) E_{g 2}+\left(\frac{Z_{2}^{2}}{Z_{\Sigma}}-Z_{2}\right) i_{v s c 2} \\
+\frac{Z_{2}}{Z_{\Sigma}} E_{g 1}-\frac{Z_{1} Z_{2}}{Z_{\Sigma}} i_{v s c 1}
\end{array}
$$

where $Z_{\Sigma}=Z_{1}+Z_{2}+Z_{l}$.

From Equations (32) and (33), one can observe that PCC voltages are influenced by both AC systems, the feedback from the current injected to the same PCC and also the controlled current from the other VSC, which is also influenced by the current from the first converter.

The complete MIMO system is depicted in Figure 8 where the transfer functions relating the different variables are obtained from Equations (32) to (33).

The closed-loop stability from the stiff grid voltages to the controlled currents is studied from the system defined by,

$$
\begin{aligned}
& i_{v s c 1}=G_{11} E_{g 1}+G_{12} E_{g 2} \\
& i_{v s c 2}=G_{21} E_{g 1}+G_{22} E_{g 2}
\end{aligned}
$$

These transfer functions relating inputs and outputs in Equation (34) are obtained by substituting Equation (32) and (33) into (29) and isolating the current cross-couplings.

Interactions are thus reflected into the converter by means of the closed-loop admittances of the system $G_{i j}$. Each of these transfer functions have been derived by considering all the possible paths and hence are influenced by all the elements which compose the network.

\section{Case study and results}

The system composed by two converters, two AC networks and a transmission line from Figure 1 is studied. The different components are substituted by their respective models developed in section 2. The MIMO system presented in section 3 is developed by substituting the equivalent models of the VSC admittance, AC network and transmission line impedances in Equations (32) and (33). Transfer functions relating the different inputs and outputs in Equation (34) are derived. Then the interactions and degree of coupling between both subsystems are evaluated by means of studying the response of the closed-loop admittance $G_{i i}$. The parameters which have an influence in the coupling between both subsytems, i.e., line and grid impedances are changed and the closed-loop response of the coupled system is compared with the closedloop response of the converter operating independently, i.e., by neglecting the parallel coupling as in Equation (28).

The complete system is expressed in $d q$ frame. Thus all the variables which appear in the MIMO system are related to the same components of the $d q$ matrices. Each of the matrix terms must be handled separately. Only the results of the $d d$-components in admittances and impedances are shown.

Special focus must be paid to the range of low frequencies and within the control bandwidth since oscillations in high frequencies are damped enough by the inherent high impedance in this range and the efficient rejection capability of the VSC to disturbances above the control bandwidth.

\subsection{Control interactions between two converters}

First the effect of interactions in the response of one VSC is illustrated. It suffices to check the diagonal closed-loop transfer function of one converter, i.e., $G_{i i}$. For limiting the study and avoiding repetition, only the first subsystem is considered. However the same results can be extrapolated to the other converter. The transfer function $G_{11}$ in Equation (34), which relates the controlled current injection of the first converter to the 


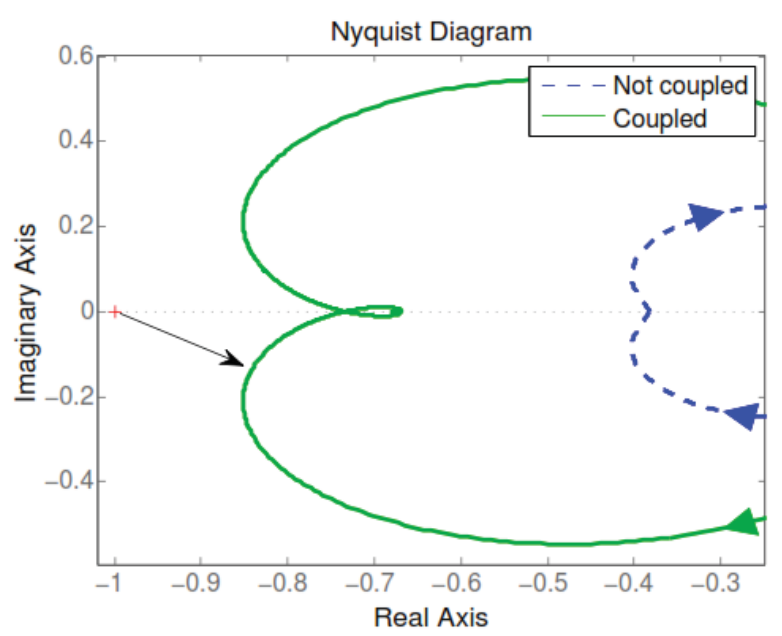

Fig. 9: Nyquist diagram of the interconnected system in solid and the system without the influence from the parallel VSC in dashed for a line length of $25 \mathrm{~km}$ and a SCR in both systems equal to 3 .

AC grid voltage of the first system, is influenced by the elements of the second subsystem such as the grid impedance and the VSC admittance of the second converter. The denominator of this transfer function, denoted as $1+L(s)$ for following common notation in literature, is,

$$
\begin{array}{r}
1+L(s)=1+Y_{v s c 1}\left[Z_{1}-\frac{Z_{1}^{2}}{Z_{\Sigma}}\right. \\
-\frac{Y_{v s c 2} Z_{1}^{2} Z_{2}^{2}}{Z_{\Sigma}\left(1-Y_{v s c 2}\left(\frac{Z_{2}^{2}}{Z_{\Sigma}}-Z_{2}\right)\right)}
\end{array}
$$

The loop transfer function, i.e., $L(s)$ in Equation (35), allows to study the closed-loop stability with the Nyquist Criterion.

For a converter independently connected the loop transfer function, and coming back to Equation (28), is $Y_{v s c 1} Z_{1}$. Therefore one sees by comparing both loop TFs the influence of the other converter's admittance $Y_{v s c 2}$.

The closed-loop stability is now studied by plotting the Nyquist curves of both loop TFs. Figure 9 depicts the Nyquist diagram of the TF in Equation (35) and the system without considering the second converter's admittance $Y_{v s c 2}$.

In the figure the curve from the loop transfer function of Equation (35), i.e., the system considering $Y_{v s c 2}$, gets closer to the critical point $(-1+\jmath 0)$. The system is not unstable due to the curve is not encircling the critical point. However this distance, depicted with an arrow in the figure, gives an idea of the relative stability. This distance indicates the peak of the sensitivity function and hence how prone the system is to instabilities under uncertainties. In other words, this measure of relative stability indicates that the system is closer to be unstable given a variation in the parameters of the plant.

As seen by the difference between the critical point and both curves, the fact of neglecting the influence of the other converter will lead to a very significant difference in the

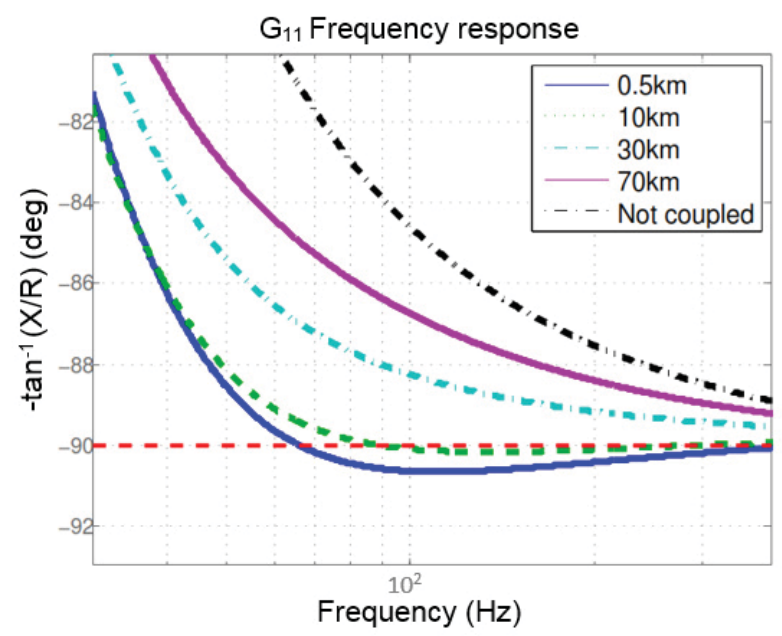

Fig. 10: Phase angle of closed-loop admittances $G_{11}$ for a line length of $0.5,10,30$ and $60 \mathrm{~km}$, for $\mathrm{P}=1 \mathrm{GW}$ and $\mathrm{SCR}=3$.

stability assessment.

\subsection{Impact of transmission line length}

Now the effect of the transmission line is addressed. This is the element which interconnects both VSCs and hence coupling the dynamics of both parallel systems.

First the diagonal closed-loop admittances $G_{11}$ for the different transmission line lengths are calculated. Figure 10 shows the results for the transmission lines. The passivity in the system is studied from the phase plot of these TFs. In the figure a new negative-conductance region appears in the range of frequencies between 55 and $250 \mathrm{~Hz}$ for the system interconnected by a $0.5-\mathrm{km}$ line. This negative region does not appear in the original admittance $Y_{v s c 1}$. And since the line and grid impedance are passive systems, the negative conductance can only be caused by the other VSC. These region is significantly damped for the $10-\mathrm{km}$ line and does not appear in the rest of cases neither in the closed-loop response neglecting the coupling. Then parallel connection of converters leads to a new point of potential instabilities around the control bandwidth.

The last results and evaluation based on the passivity of the system are compared with another frequency-domain analysis. As done before, the Nyquist curves for the different line lengths and for the system neglecting the influence of the second converter are plotted. Figure 11 shows the results. Smaller line lengths have the Nyquist curve $L(\jmath \omega)$ closer to the critical point. Therefore this indicates that the shorter the line, the more deteriorated relative stability is. However variations in the line length do not have a significant impact on the relative stability as seen by the proximity between the different Nyquist curves. In case of neglecting the influence of the parallel-connected converter, the Nyquist curve is 


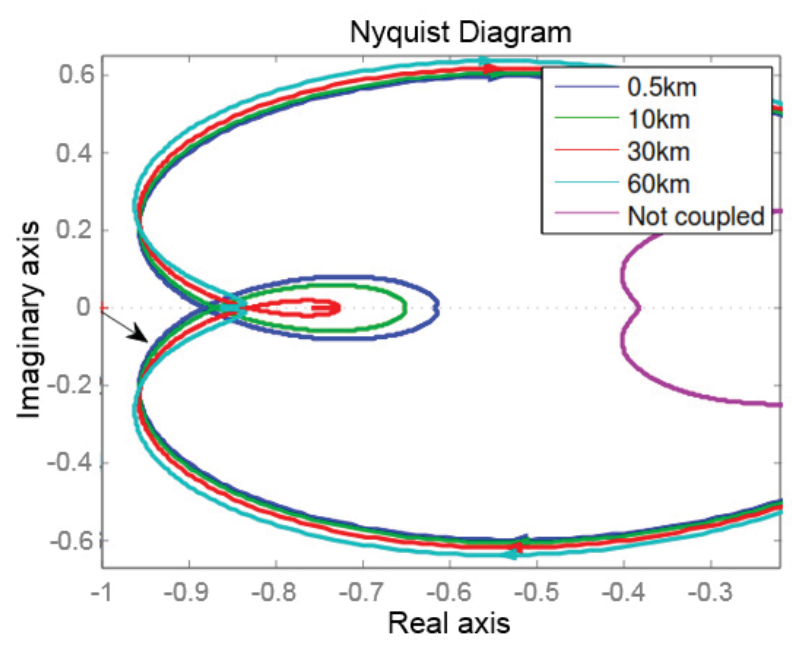

Fig. 11: Nyquist curves for the different loop TFs with variable line length.

further from the rest of curves. This fact gives an idea of the consequences of simplifying by neglecting the impact of a parallel-connected VSC in the relative stability of one converter.

To sum up, both theories, the Nyquist Criterion and the passivity of the system, indicate the relative stability deterioration and hence a higher sensitivity under uncertainty. However the differences between the studied transmission lines are not significant. This is due to the simplified model consisting of two AC systems and one line interconnecting both. Since grid impedances are the main limiting factor to the propagation of dynamics, the line will conduct all the possible disturbances. Also its magnitude is significantly smaller than the magnitude of the grid impedances.

\subsection{Short circuit capacity}

Finally the last element which compose the model, the grid impedance, is studied. The AC system strength is a characteristic which plays a significant role in the propagation of dynamics. Stiffer networks easily absorb distortions at the node and thus do nor lead to stability issues in the VSC connected to the grid neither propagate the dynamics to other points of the system.

Its impact on the influence between converters is addressed by varying the grid impedance magnitude. In this case, only the SCR from the grid to which the second converter is connected is modified.

Figure 12 shows the phase angle under different network impedances. Systems connected to low SCRs approach closer to the non-passive region. Specifically the case with the highest grid impedance, SCR equal to 2.5, lags over -90 and hence indicates a region of negative damping. As in the previous sections, the problem is also analysed with the Nyquist criterion. The Nyquist curve of the loop transfer function

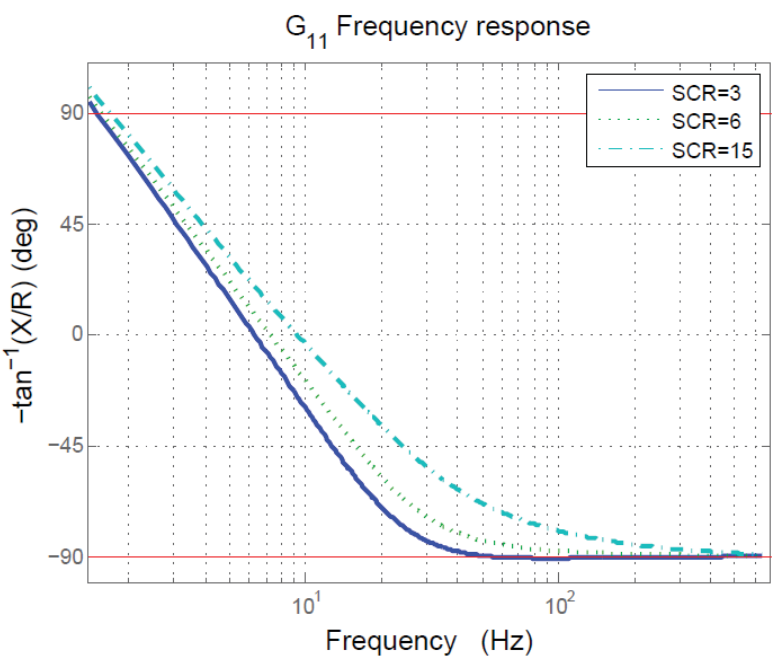

Fig. 12: Phase angle of $G_{11}$ closed-loop admittances for SCRs equal to $2.5,7.5$ and 25 for a line of $25 \mathrm{~km}$ and $\mathrm{P}=1 \mathrm{GW}$.

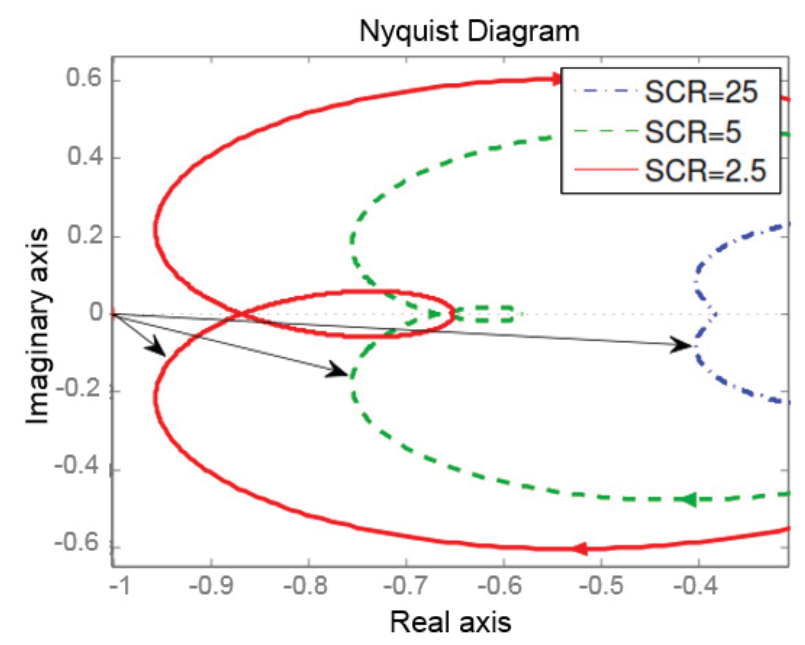

Fig. 13: Nyquist curves for SCRs equal to 2.5, 7.5 and 25.

in the denominator is plotted for different SCRs. Figure 13 shows the results. A large and significant difference in the distance from the critical point to the Nyquist curve is seen. Systems with higher grid impedance get closer to the critical point and are thus more sensitive to uncertainties. Therefore interactions between converters are highly dependant on the strength of the system.

\section{Conclusions}

Interactions between voltage source converters connected at different points in the grid are studied in this paper. The focus is on the methodology to evaluate the stability in such a coupled system. An increasing number of converters will be integrated to the system in the future and unexpected 
interactions between them might lead to stability issues. The importance of this study results from the need of evaluating the influence of these interactions in the stability assessment and the impact of the electrical distance between devices. The assessment by means of EMT studies of such a large, complex and with a wide range of scenarios makes it an arduous task. However frequency-domain methods allow to detect potential points of instability and its expansion is not as complex as in time-domain methods.

The paper challenges the study of the closed-loop response of a system with two converters and evaluates the role of, in this case, the transmission line and the impact of the AC system strength. The impedance-based approach is expanded for including both converters and stability is studied from the closed-loop transfer functions of the complete system.

Simulation results reveal the proneness to instability of one converter due to the influence of the other VSC. The relative stability of one converter is deteriorated due to interactions from the other converter. Results show that shorter lines and higher grid impedances impose limitations in the stability margins and the system is more sensitive under uncertainties. In the studied model, grid impedances have larger impacts on the influence between converters whilst the transmission line has a minor effect. Therefore interactions between converters are mainly dependant of the strength of the system in such a topology of the network and the need of considering all the dynamics is more important in weaker systems.

\section{Acknowledgements}

The research leading to these results has received funding from the People Programme (Marie Curie Actions) of the European Unions Seventh Framework Programme FP7/2007-2013/ under REA grant agreement no. 317221, project title MEDOW. Jef Beerten is funded by a post-doctoral fellowship from the Research Foundation Flanders (FWO-Vlaanderen).

\section{References}

[1] L. Harnefors, M. Bongiorno, S. Lundberg. "InputAdmittance Calculation and Shaping for Controlled Voltage-Source Converters", IEEE Trans. on Ind. Elec., vol.54 n.6, pp. 3323-3334, (2007).

[2] J. Enslin, P. Heskes. "Harmonic interaction between a large number of distributed power inverters and the distribution network", IEEE Trans. on Power Electronics, vol.19 no.6, pp. 1586-1593, (2004).

[3] P. Brogan. "The stability of multiple, high power, active front end voltage sourced converters when connected to wind farm collector systems", EPEC Proceedings, (2010).

[4] Ł. Kocewiak, J. Hjerrild, C. Leth Bak. "Wind turbine converter control interaction with complex wind farm systems", IET Renewable Power Generation, vol.7 no.4, pp. 380-389, (2013).

[5] K. Alawasa, Y. Abdel-Rady, W. Xu. "Modeling, analysis and suppression of the impact of full-scale wind-power converters on subsynchronous damping", IEEE Systems Journal, vol.7 no.4, pp. 700-712, (2013).

[6] C. Wan, M. Huang, C.K. Tse, X. Ruan. "Stability of interacting grid-connected power converters", Journal of Modern Power Systems and Clean Energy, Springer, vol.1 no.3, pp. 249-257, (Dec. 2013).

[7] L. Shen, M. Barnes, J.V. Milanović, K.R.W. Bell, M. Belivanis. "Potential Interactions between VSC HVDC and STATCOM ", $18^{\text {th }}$ PSCC in Wroclaw, Poland, (2014).

[8] X. Wang, F. Blaabjerg, W. Wu. "Modeling and Analysis of Harmonic Stability in an AC Power-ElectronicsBased Power System", IEEE Trans. on Power Electronics, vol.29 no.12, pp. 6421-6432, (Dec. 2014).

[9] J. Sun. "Impedance-Based Stability Criterion for GridConnected Inverters", IEEE Trans. on Power Electronics, vol.26 no.11, pp. 3075-3078, (2011).

[10] M. Cespedes, J. Sun. "Impedance modeling and analysis of grid-connected voltage-source converters", IEEE Trans. Power Electronics, vol.29 no.3, pp. 1254-1261, (Mar. 2014). 